\title{
Case Report \\ Canine distemper virus, atypical Toxoplasma gondii, and Neospora caninum co-infection, in a dog with neurological signs from Argentina
}

\author{
Gabriela C. Postma ${ }^{1 *}$, Andrea Dellarupe ${ }^{2,3}$, Nicolás Streitenberger ${ }^{4}$, Ana Bratanich ${ }^{5}$, María C. \\ Venturini $^{2}$, Leonardo Minatel $^{1}$ \\ ${ }^{1}$ Universidad de Buenos Aires, Facultad de Ciencias Veterinarias, Cátedra de Patología, Chorroarín 280, C1427CWO, Buenos Aires, Argentina. \\ ${ }^{2}$ Laboratorio de Inmunoparasitología (LAINPA), Facultad de Ciencias Veterinarias, Universidad Nacional de La Plata, 60 y 118 (1900), La Plata, \\ Argentina. \\ ${ }^{3}$ Consejo Nacional de Investigaciones Científicas y Técnicas (CONICET). \\ ${ }^{4}$ Laboratorio de Patología Especial Veterinaria (LAPEVET), Facultad de Ciencias Veterinarias, Universidad Nacional de La Plata, Argentina. \\ ${ }^{5}$ Universidad de Buenos Aires, Facultad de Ciencias Veterinarias, Cátedra de Virología, Buenos Aires, Argentina. \\ *Corresponding author: Chorroarín 280, C1427CWO, Buenos Aires, Argentina. Phone: +54114580-2821. E-mail: gpostma@ fvet.uba.ar
}

Submitted May, 26 $6^{\text {th }} 2018$, Accepted August, $11^{\text {th }} 2018$

\begin{abstract}
The occurrence of combined infections by Toxoplasma gondii, Neospora caninum and Canine distemper virus (CDV) in domestic dogs and wildlife animals has not been frequently reported, and the histopathological findings were not exhaustively described. The objective of this study was to report a co-infection of CDV, T. gondii and N. caninum in a dog with neurological signs, as well as the molecular characterization of the protozoa involved. A young street dog was rescued with neurological clinical signs and died spontaneously. A complete necropsy was performed. Tissues were collected and fixed for histopathological evaluation. Additionally, sections of the central nervous system (CNS) and heart were assayed by immunohistochemistry (IHQ) for T. gondii and N. caninum. Sample of brain tissue was analyzed by PCR and nPCRRFLP for $T$. gondii genotyping. Spleen was used for detection of CDV by RT-PCR. Gross lesions were not observed, with the exception of the lung. Microscopically, a severe necrosuppurative meningoencephalitis with vasculitis, tachyzoites and bradyzoites of $T$. gondii and $N$. caninum were found. Demyelination was also evident, associated with eosinophilic intranuclear inclusion bodies within astrocytes. CDV was PCR positive while both parasites were presented PCR and IHQ positive results. Molecular characterization of $T$. gondii was reported as atypical \#14 (likely). To our knowledge, this is the first report of genetical identification of $T$. gondii obtained from the brain of a naturally infected dog in Argentina. The results emphasize the importance of different techniques as diagnostic tools to enhance the detection of causative agents in cases of fatal encephalitis.
\end{abstract}

Key words: Toxoplasma gondii, Neospora caninum, canine distemper virus, genotyping.

\section{Introduction}

Concurrent infections by Canine distemper virus (CDV), Toxoplasma gondii and Neospora caninum are not frequently reported. However, it is well known that clinical manifestation of apicomplexan protozoan parasites in dogs could occur under immunosuppression, such as that caused by CDV, due to the effect of this viral pantropic infection on lymphocytes and macrophages $(4,13)$. Some authors have reported the occurrence of combined infections by one of these types of parasites and CDV in domestic dogs $(9,15)$, and wildlife animals $(5,11,18)$, but the histopathological findings were absent or not exhaustively described. 
Toxoplasma gondii in dogs is considered an opportunistic agent that can parasitize a wide variety of cell types in the body, although the encephalic form of toxoplasmosis is most likely to occur under immunosuppression (22). Molecular genotyping studies have shown a highly clonal population structure for $T$. gondii made up of three clonal lineages, designated types I, II and III, which differ in their virulence in mice (10). However, recombinant or non-clonal pattern, namely atypical, have been detected in many countries, and are overrepresented in South America $(1,16,17)$. Little is known about genotyping of $T$. gondii in domestic and wild animals, even humans, in Argentina (3). The objective of this study was to report a co-infection of CDV, T. gondii and $N$. caninum in a dog with neurological signs, as well as to analyze the molecular characterization of the protozoa involved.

\section{Case Report}

A young street dog, probably under a year old, was submitted for necropsy to the Pathology Veterinary Service of Buenos Aires University, Buenos Aires, Argentina. The dog was rescued from the street with clinical signs of depression, reduce appetite, and poor general condition. During the hospitalization, it manifested nervous tics, delirium and, when placed in lateral recumbency, paddling movements, prior to dying spontaneously. A complete necropsy was performed. Tissues were collected and fixed in $10 \%$ neutral buffered formalin solution for histopathological evaluation. Additionally, sections of the central nervous system (CNS) and heart were assayed by immunohistochemistry (IHQ) for T. gondii and $N$. caninum. Samples of brain tissue and spleen were stored at $-20{ }^{\circ} \mathrm{C}$ to further molecular test of apicomplexan coccidian and CDV, respectively. DNA was extracted from brain tissue using a commercial kit (Wizard® Genomics DNA Purification Kit, Promega, USA) according to the manufacturer's protocol at LAINPA. Polymerase chain reaction (PCR) was performed using specific primers for T. gondii (Tox5-Tox8) and N. caninum (Np6+-Np21+), as described previously (14). Each amplification routine was conducted with the positive control (DNA from $T$. gondii RH strain and $N$. caninum Nc-1 strain, respectively), a negative control (control process sample DNA) and a no template control (NTC). Genotyping of $T$. gondii sample was performed by nPCRRFLP using ten genetic markers, as described previously (20). DNA from RH, Me49 and NED T. gondii strains were used as genotype I, II, and III controls, respectively. The result was compared to the ToxoDB genotyping database (www.Toxodb.org) and designated with the matching genotype \#. Brain, cerebellum and heart samples were submitted for IHQ to LAPEVET. $T$. gondii and $N$. caninum immunostaining were performed using the conventional peroxidase technique with the LSAB2 $®$
System HRP (Dako, USA) according to the manufacturer's instructions. A rabbit $T$. gondii polyclonal anti-serum (gently provided by Dr. María Cecilia Venturini) and a rabbit $N$. caninum polyclonal anti-serum (gently provided by Dr. Lucía M. Campero) were used as primary antibodies at 1:3000 and 1:1200 dilution (Da Vinci Green Diluent, Biocare Medical, USA), respectively, and were incubated overnight at $4^{\circ} \mathrm{C}$. Lung tissue from a $T$. gondii naturally infected goat, and heart tissue from an $N$. caninum experimentally infected mouse were used as positive tissue controls. Furthermore, each tested tissue was incubated with commercial diluent as negative reagent controls. RNA was extracted from spleen tissue with Trizol (Invitrogen, USA) at the Department of Virology, Buenos Aires University. Approximately $1 \mu \mathrm{g}$ of RNA was used for cDNA synthesis with a specific reverse primer (NP2) targeting the nucleoprotein (NP) gene (7). cDNA synthesis was performed with a commercial kit (Improm System, Promega, USA), following manufacturer's instructions. Five $\mu \mathrm{l}$ of the reaction was used for a conventional PCR reaction employing NP1 Foward (ACAGGATTGCTGAGGACCTAT) and NP2 Reverse (CAAGATAACCATGTACGGTGC) primers. Internal controls were run in parallel to evaluate RNA integrity using primers that target a canine housekeeping gene GAPDH (7), $\quad$ GAPDH $\quad$ Foward (GCCAAAAGGGTCATCATCTC), and GAPDH Reverse (GGCCATCCACAGTCTTCT), following the same PCR conditions as described for the NP-specific primers.

Grossly, the cadaver was in poor body condition and it had a severe degree of dehydration. A bilateral mucopurulent ocular discharge was observed. There was a dark red area of consolidation in the accessory lobule of the right lung. The consistency of the rest of the lung was rubbery. Gross lesions were not observed in major organs and the CNS. Microscopically, multifocal and widely distributed in the brain, there were scattered areas of severe inflammation and necrosis fairly well circumscribed, characterized by disruption and loss of the normal architecture, with the replacement of the parenchyma by moderate amounts of necrotic cellular and karyorrhectic debris, edema, neutrophils, lymphocytes, and plasma cells. The cerebellum was severely affected, with necrosis and loss of cerebellar grey matter extending into the adjacent white matter (Fig. 1); while cerebral lesions were seen predominantly in the grey matter of the cortex, and around the ependymal cells, lining the ventricles. Nevertheless, necrosis and inflammation were present in all sections of the brain, including mesencephalon, pons and the medulla oblongata. Blood vessels within the necrotic foci showed vasculitis (Fig. 1); and those adjacent to necrotic areas had distended perivascular spaces, surrounded by moderate numbers of lymphocytes, plasma cells and fewer macrophages (perivascular cuffing). The same type of inflammatory infiltrate was observed in the meninges. 
Within and adjacent necrotic foci there were some 2-4 $\mu \mathrm{m}$ in size, extracellular, round to oval, basophilic organisms (Fig. 2), compatible with tachyzoites of protozoan parasites, which were occasionally found within endothelial cells. Few clusters of $15-40 \mu \mathrm{m}$ in diameter, presumed cyst with bradyzoites, were noted within and around the lesions (Fig. 3).

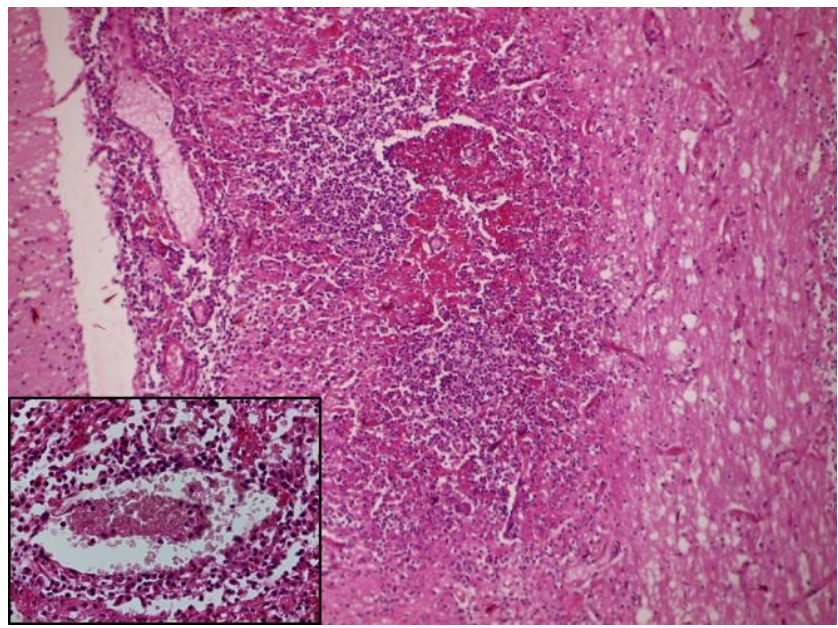

Figure 1. Cerebellum cortex and white matter. Note focal necrotizing lesion with severe inflammation, and vasculitis (inset) (HE, 100x).

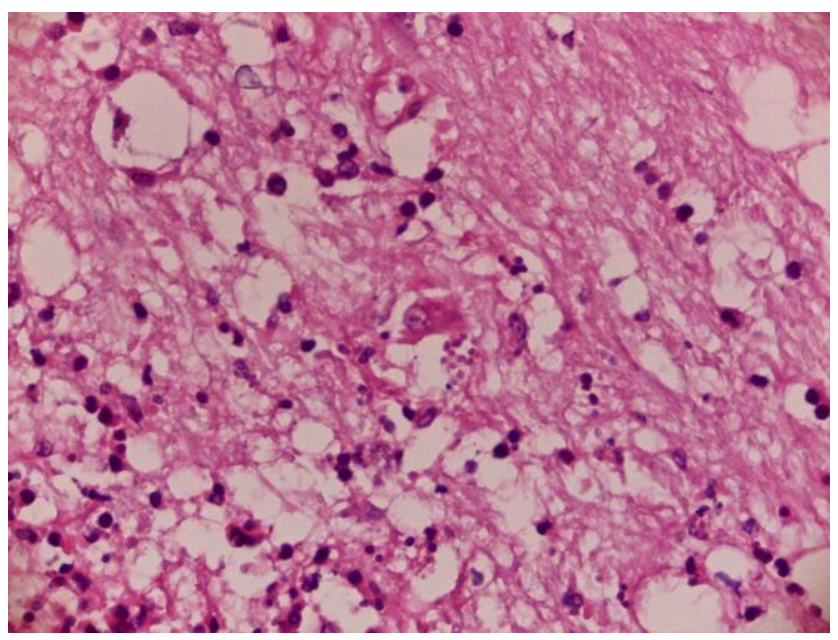

Figure 2. Thalamus cortex. Note few basophilic organisms compatible with tachyzoites of protozoan parasites, adjacent to a gemistocytic astrocyte, mild inflammation and spongiosis (HE, 630x).

In not affected periventricular areas, some cysts with bradyzoites were found. Both tachyzoites and cysts stained strongly for $T$. gondii and $N$. caninum by IHC. Randomly dispersed throughout less affected areas, there were small aggregates of glial cells (multifocal gliosis). Widespread vacuolization of white matter tracts (spongiosis) was also evident, without any substantial amount of inflammation. Sporadic foci of axonal swelling and spheroids, and a moderate number of Gitter cells (malacia areas) were noted. Demyelination was most severe in the cerebellum, while other parts of the brain were less severely affected. Moderate eosinophilic intranuclear inclusion bodies, surrounded by a narrow halo with margination of chromatin, where found within astrocytes from cerebellum (Fig. 4).

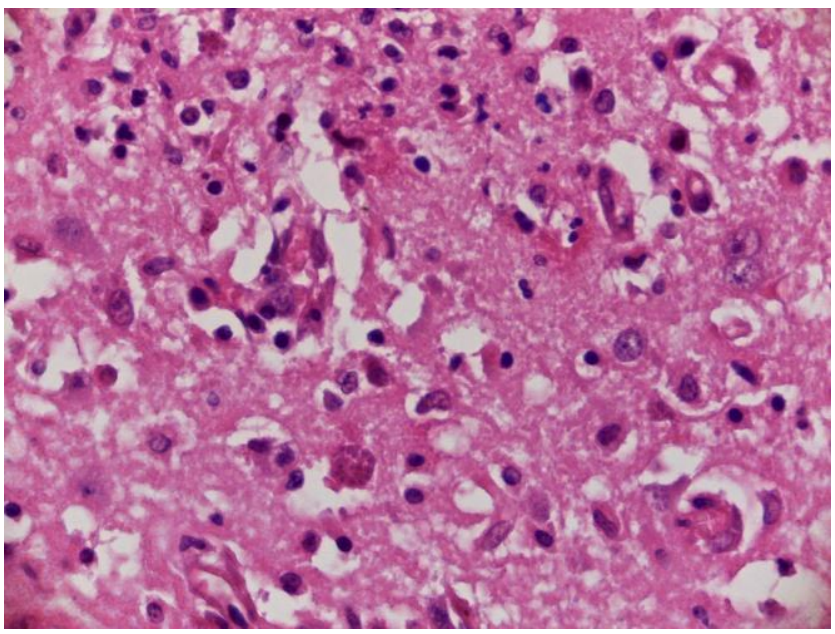

Figure 3. Parietal lobe (cortex). A cluster of bradyzoites of protozoan parasites, adjacent to inflammatory foci (HE, 630x).

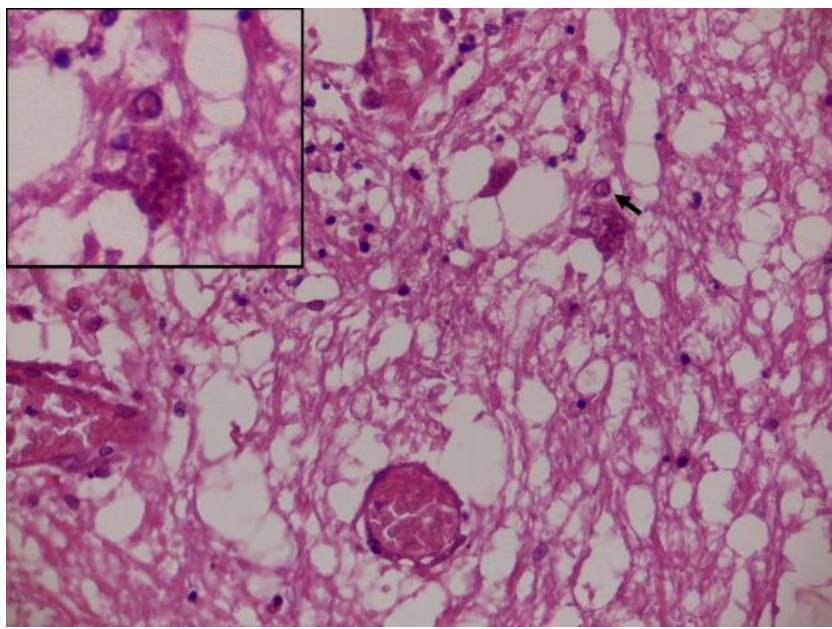

Figure 4. Cerebellum white matter. Intranuclear acidophilic inclusion body within an astrocyte (arrow). Note intense demyelination around, and a cluster of tachyzoites of protozoan parasites below the inclusion body (inset) (HE, 400x).

Other significant histopathological alterations included interstitial pneumonia associated with suppurative exudate in bronchioles and alveoli and attenuation of bronchiolar epithelium. Intracytoplasmic eosinophilic inclusion bodies were found in large quantities within parietal cells of the gastric epithelium. Palatine tonsils and lymph nodes showed lymphocytic depletion and 
hyperplasia of histiocytic cells. Additionally, there was a small multifocal nonsuppurative myocarditis. Furthermore, cysts of $T$. gondii were identified by IHC in not affected areas of the myocardium.

The DNA extracted from CNS of the dog was positive for $T$. gondii and N. caninum by PCR. T. gondii PCR-positive sample was successfully genotyped by nPCR-RFLP, with most markers showing a type III restriction pattern, except for SAG1 and C29-2 (type I) and was identified as \#14 (likely). The quantity of $N$. caninum DNA was not enough to carry out the genetic characterization. Amplicons of 299 bp CDV NP and 229 bp canine GAPDH specific fragments were successfully amplified from the spleen material.

\section{Discussion}

The results of this investigation revealed the participation of CDV, T. gondii and $N$. caninum in brain lesions, accessed by histopathology, IHC, and molecular techniques. This molecular approach contributes to the diagnosis and is recommended for the differentiation of apicomplexan protozoa within tissue samples (21). Although there are morphologic differences between $T$. gondii and $N$. caninum, immunohistochemistry technique or, not so frequently used, the electron microscopy observations, are required to distinguish those protozoa organisms microscopically (6). The neurological clinical syndrome linked to any of the three agents is indistinguishable (22). The immunosuppressive effects of CDV would have facilitated the simultaneous occurrence of these opportunistic agents by reactivation of latent inactive cysts in neural tissue, or by exacerbation of a present infection (13). Clinical neurological signs are more severe, and the prognosis is poorer in animals suffering coinfections (13). The presence of tachyzoites associated with the lesions points out to a lack of host immune control of parasite multiplication. The severity of cerebellum observed in this study corroborates with some reports that describe necrotizing cerebellitis as a significant lesion associated with Neospora encephalitis in dogs $(8,12)$. On the other hand, demyelination plus intranuclear acidophilic inclusion bodies in astrocytes and gastric epithelium are histopathological findings for CDV infection (4). Microscopic periventricular involvement found in this work probably arises from the distribution of CDV through the cerebrospinal fluid (4). To our knowledge, this is the first report with the presence of $T$. gondii in the brain sample of a naturally infected dog, in Argentina. A recent report analyzed the population structure of $T$. gondii in 39 samples of animals and humans from Argentina (3). Twenty-nine samples were classified according to ToxoDB as non-clonal with a predominance of type III alleles, in concordance with this case report and others from South America $(16,17)$. The genotype demonstrated in this work
(\#14 likely) was also found in a human and a wildlife animal (Bennett's Wallaby, Macropus rufogriseus

) from Buenos Aires, Argentina (2, 3), and chickens (Gallus gallus domesticus) from Misiones, Argentina (Pardini et al., unpublished data). Since both humans and dogs are exposed to the same source of infection represented by the environment and alimentary habits, these investigations may help to identify common potential infection sources (19). Our result provides new genetic information about $T$. gondii in dogs and has implications for better understanding of the genetic population diversity and epidemiology of $T$. gondii in Argentina. To obtain more accurate information about the genetic characteristics of Toxoplasma gondii and $N$. caninum in dogs from Argentina, further studies with samples from different regions of the country should be carried out. Histopathological, immunohistochemical and molecular findings in our report highlight the importance of combining different techniques as diagnostic tools to enhance the detection of causative agents in cases of fatal encephalitis and allows to differentiate these diseases from other neurological diseases in dogs.

\section{Acknowledgments}

We thank Dr. María Alejandra Quiroga for her academic contribution.

This research did not receive any specific grant from funding agencies in the public, commercial, or notfor-profit sectors

\section{References}

1. Ajzenberg D, Banuis AL, Su C, Dumetre A, Demar M, Carme B, Darde ML. Genetic diversity, clonality and sexuality in Toxoplasma gondii. Int J Parasitol. 2004;34:1185-96.

2. Basso W, Venturini MC, Moré G, Quiroga MA, Bacigalupe D, Unzaga JM, Larsen A, Laplace R, Venturini L. Toxoplasmosis in captive Bennett's wallabies (Macropus rufogriseus) in Argentina. Vet Parasitol. 2007;144:157-61.

3. Bernstein M, Pardini L, Moré G, Unzaga JM, Chunlei S, Venturini MC. Population structure of Toxoplasma gondii in Argentina. Infect Genet Evol. 2018;65:72-9.

4. Caswell JL, Williams KJ. Respiratory system. In: Maxie MG, editor. Jubb, Kennedy and Palmer's Pathology of Domestic Animals. 6th ed. St. Louis: Elsevier; 2016.

5. Diters RW, Nielsen SW. Toxoplasmosis, distemper and herpesvirus infection in a skunk (Mephitis mephitis). J Wildl Dis. 1978;14:132-6.

6. Dubey JP, Lappin MR. Toxoplasmosis and Neosporosis. In: Greene CE, editor. Infectious disease of the dog and cat. 4th ed. Georgia: Elsevier; 2012. 
7. Frisk AL, König $\mathrm{M}$, Moritz $\mathrm{A}$, Baumgärtner W. Detection of canine distemper virus nucleoprotein RNA by reverse transcription-PCR using serum, whole blood, and cerebrospinal fluid from dogs with distemper. J Clin Microbiol. 1999;37:3634-43.

8. Garosi L, Dawson A, Couturier J, Matiasek L, de Stefani A, Davies E, Jeffery N, Smith P. Necrotizing cerebellitis and cerebellar atrophy caused by Neospora caninum infection: magnetic resonance imaging and clinicopathologic findings in seven dogs. J Vet Int Med. 2010;24:571-8.

9. Headley SA, Alfieri AA, Torres Tomasi Fritzen J, Garcia JL, Weissenböck H, Da Silva AP, Bodnar L, Okano W, Alfieri AF. Concomitant canine distemper, infectious canine hepatitis, canine parvoviral enteritis, canine infectious tracheobronchitis, and toxoplasmosis in a puppy. J Vet Diagn Invest. 2013;25:129-35.

10. Howe DK, Sibley LD. Toxoplasma gondii compromises three clonal lineages: correlation of parasite genotype with human disease. J Infect Dis. 1995;172:1561-6.

11. Lemberger KY, Gondim LF, Pessier AP, McAllister MM, Kinsel MJ. Neospora caninum infection in a free-ranging racoon (Procyon lotor) with concurrent canine distemper virus infection. J Parasitol. 2005;91:960-1.

12. Lorenzo V, Pumarola M, Sisó S. Neosporosis with cerebellar involvement in an adult dog. J Small Anim Pract. 2002;43:76-9.

13. Miller AD, Zachary JF. Nervous system. In: Zachary JF, editor. Pathologic Basis of Veterinary Disease. 6th ed. Illinois: Elsevier; 2017.

14. Moré G, Pardini L, Basso W, Machuca M, Bacigalupe D, Villanueva MC, Shares G, Venturini MC, Venturini L. Toxoplasmosis and genotyping of Toxoplasma gondii in Macropus rufus and Macropus giganteus in Argentina. Vet Parasitol. 2010;169:5761.

15. Moretti L, Da Silva AV, Ribeiro MG, Paes AC, Langoni H. Toxoplasma gondii genotyping in a dog co-infected with distemper virus and erlichiosis rickettsia. Rev Inst Med Trop S Paulo. 2006;48:35963.

16. Pena HF, Gennari SM, Dubey JP, Su C. Population structure and mouse-virulence of Toxoplasma gondii in Brazil. Int J Parasitol. 2008;38:561-9.

17. Rajendran C, Su C, Dubey JP. Molecular genotyping of Toxoplasma gondii from Central and South America revealed high diversity within and between populations. Infect Genet. Evol. 2012,12:359-68.

18. Reed WM, Turek JJ. Concurrent distemper and disseminated toxoplasmosis in a red fox. J Am Vet Med Assoc. 1985;187:1264-5.

19. Silva LA, Andrade RO, Carneiro AC, Vitor RW. Overlapping Toxoplasma gondii genotypes circulating in domestic animals and humans in Southeastern Brazil. PLoS One. 2014;9:e90237.

20. Su C, Zhang X, Dubey JP. Genotyping of Toxoplasma gondii by multilocus PCR-RFLP markers: a high resolution and simple method for identification of parasites. Int J Parasitol. 2006;36:841-8.

21. Su C, Shwab EK, Zhou P, Zhu XQ, Dubey JP. Moving towards an integratin approach to molecular detection and identification of Toxoplasma gondii. Parasitol. 2010;137:1-11.

22. Uzal FA, Plattner BL, Hostetter JM. Alimentary system. In; Maxie MG, editor. Jubb, Kennedy and Palmer's Pathology of Domestic Animals. 6th ed. St. Louis: Elsevier; 2016. 